\title{
Study of parallel-connected PPD readout scheme
}

\author{
Daichi Naito* \\ Kyoto Univ. \\ E-mail: d.naito@scphys.kyoto-u.ac.jp
}

In the $\mathrm{K}^{O} \mathrm{TO}$ experiment, we plan to use a plastic scintillator as a veto counter; its role is to reject various K-decay modes involving charged particles. This counter, called CV(Charged Veto), must detect an energy deposit as low as $100 \mathrm{keV}$. To realize this, we use Pixelated Photon Detector(PPD) as a photo-sensor in conjunction with wavelength shifting fibers. About 1600 channels are needed in total, and 8 PPDs outputs are connected together and are read as one to reduce the readout channels. One of the disadvantages of this readout scheme is that their output pulse becomes wider as the number of parallel-connected PPD increases. We report results of the study on this phenomena and present a method to overcome the pulse widening.

International Workshop on New Photon Detectors

June 24-26, 2009

Shinshu University Matsumoto Japan

\footnotetext{
* Speaker.
} 


\section{Introduction}

\section{1 $\mathrm{K}^{O} \mathrm{TO}$ experiment}

The $\mathrm{K}^{O} \mathrm{TO}$ experiment at the J-Parc 50-Gev PS is a dedicated experiment to search for the CP-violating rare decay mode $K_{L} \rightarrow \pi^{0} v \bar{v}$. Theoretically the mode is very clean; according to the Standard Model (SM), its amplitude is proportional to $\eta$, the complex component of the CabibboKobayashi-Maskawa matrix, and is not obscured by hadronization part. Thus any deviation from the SM prediction would mean the physics beyond SM. Experimentally, however, the mode is a challenge; this is because the SM prediction of its branching ratio is very small $\left(\sim 2.5 \times 10^{-11}\right)$, and the decay mode only involves neutral particles. We take the following strategy to reach our goal[1]. See Fig. 1 for the experimental setup. First, $\pi^{0}$ s from $K_{L}$ decay are detected by a CsI calorimeter and their energies and positions are measured. From the information, $\pi^{0}$ decay vertex as well as their momentum are calculated, and various cuts are applied to confirm the event stemming from $K_{L} \rightarrow \pi^{0} v \bar{v}$. Second, hermetic veto counters surrounding the decay volume ensure nothing but $\pi^{0}$ is produced. We note the decay volume is evacuated down to $10^{-5} \mathrm{~Pa}$ to suppress backgrounds.

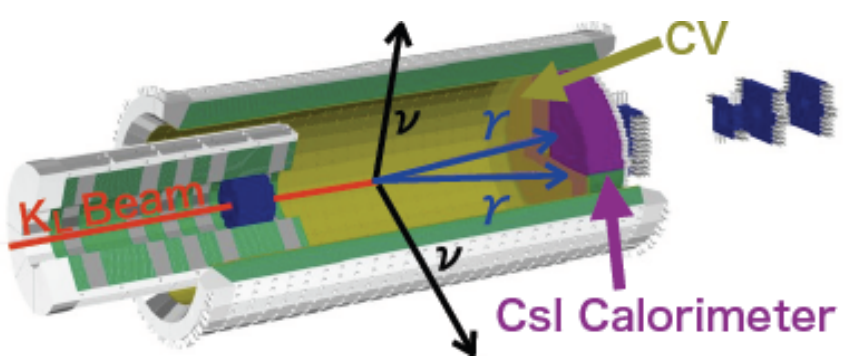

Figure 1: An illustration of the K0TO detector. Hermetic veto counters surrounding the decay volume are shown. A set of charged veto counters (CV), discussed in this paper, is located in front of the calorimeter.

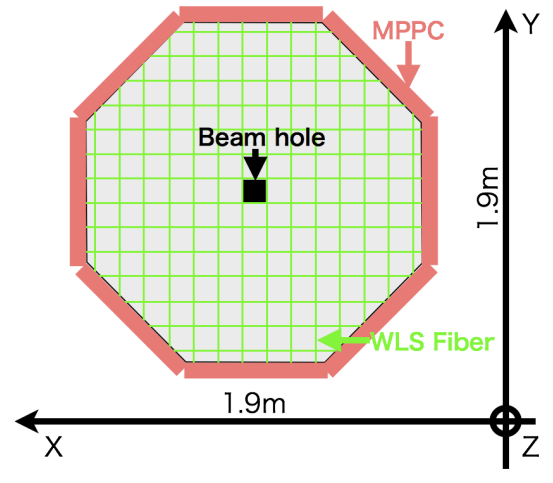

Figure 2: View of CV from the upstream of the beam.

\subsection{Charged Veto(CV)}

$\mathrm{CV}$ is a plastic scintillation counter placed in front of the CsI calorimeter. Its main role is to detect and veto charged particles from various $K_{L}$ decay modes. To achieve good efficiency without signal loss, CV must detect an energy deposit as low as $100 \mathrm{keV}$ and must have time resolution less than $2 \mathrm{~ns}$. CV must be made as thin as possible in order to reduce backgrounds caused by neutron interactions; they produce $\pi^{0}$ or $\eta$, among others, which might be misidentified as a signal. Actually, CV consists of two modules, each being octagonal-shaped $3 \mathrm{~mm}$ thick planes(Fig. 2). One module is set in front of CsI, and the other at $25 \mathrm{~cm}$ upstream of CsI. With this design, we expect the backgrounds mentioned above are very small, as shown in Table 1 . We plan to use wavelength shifting fibers to transmit lights from scintillators, and PPDs as photo sensors. The merit of using PPDs is that they have high detection efficiency and excellent single photon resolving power. In addition, since PPDs are physically small, we can put them near the detector to reduce the attenuation loss of light yields in the fiber. We compared basic properties of PPDs 
and decided using multi-pixel photon counters (MPPC) manufactured by Hamamatsu Photonics[2]. We used MPPCs with 1.3-mm-square sensitive area and 50 $\mu \mathrm{m}$ pixel size. About 1600 channels are needed in total, and 8 MPPC outputs are connected together to reduce the readout channels (Fig. $3)$. One disadvantage of this readout scheme is increase of dark count noise. We measured actual dark counts as a function of temperature and estimated the acceptance loss, the loss of signal by accidental coincidence with CV dark counts. From the results, shown in Fig. 4, we conclude that it is necessary to keep the temperature below 5 deg to make the loss negligible. There is another disadvantage in our case, which will be discussed in the next section.

\begin{tabular}{|l|r|}
\hline & \# of event \\
\hline signal & 1.41 \\
\hline charged BG & 0.2 \\
\hline$\pi^{0} \mathrm{BG}$ & 0.16 \\
\hline$\eta \mathrm{BG}$ & 0.07 \\
\hline
\end{tabular}

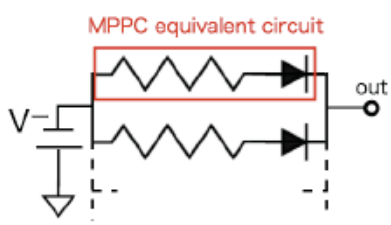

Table 1: Background estimation, through Figure 3: A schematic diagram of the CV readout. MPPCs are 3 snowmass years run time. connected in parallel and supplied voltage from same source.

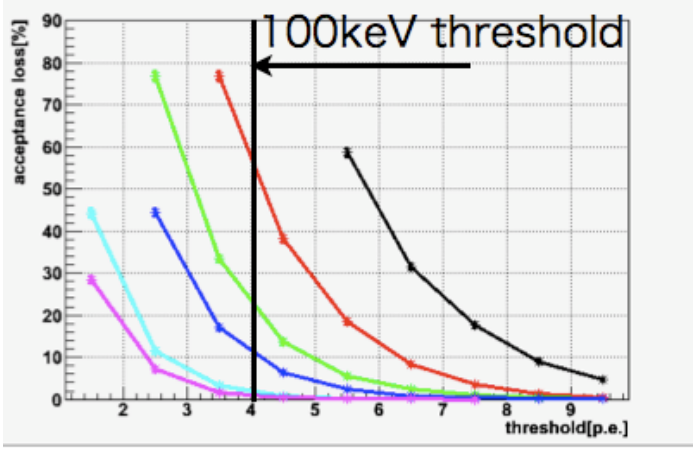

Figure 4: Dependence of the acceptance loss on temperature. Bias voltage of MPPCs is same at each temperature $(\Delta \mathrm{V}=1.2) .6$ lines, starting from the right, show the results of $25^{\circ} \mathrm{C}, 20^{\circ} \mathrm{C}, 15^{\circ} \mathrm{C}, 10^{\circ} \mathrm{C}, 5^{\circ} \mathrm{C}$, $0^{\circ} \mathrm{C}$, respectively. A vertical line indicates the threshold equivalent to $100 \mathrm{keV}$. The loss is about $55 \%$ at $20^{\circ} \mathrm{C}$ and we need cool MPPCs less than $5^{\circ} \mathrm{C}$.

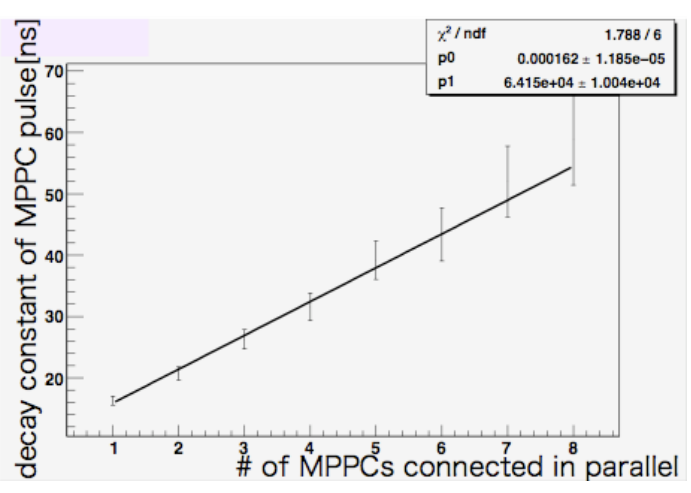

Figure 5: correlation between number of MPPCs connected in parallel and decay constant of MPPCs.

\section{2. parallel-connected MPPC readout scheme}

\subsection{Pulse width of MPPCs connected in parallel}

We found when MPPCs are read out in parallel, their effective output pulse widths become wider. Fig. 5 shows the decay time, defined as the time between the peak and its 1/e point, as a function of number of connected MPPCs. This pulse widening phenomenon can be explained by a model which takes into account the effect of spectator pixels, the pixels in which no avalanche take place. Fig. 6 shows an equivalent circuit for such a model[3]. The decay time constant is then 
represented by

$$
T_{\text {decay }}=(N-n) \times C \times R_{L}+((N-n) \times C) \times(R /(N-n))+n \times C \times R_{L}
$$

where $C$ and $R$ are, respectively, capacitance and quenching resistance of one pixel, and $N(n)$ denotes the number of total (avalanched) pixels. We note in Eq.(2.1), the first and second term represents the effect of spectator pixels while the third the effect of avalanched pixels. In order to confirm validity of this model, we measured the decay time constant with various $R_{L}$. The results, shown in Fig. 7, confirm linear dependence to $R_{L}$ as expected. If the pulse width becomes wider, pulse pile-up probability increases and time distribution of the rising edge becomes broad, and these effect may make timing resolution worse. Next section, we report the method to reduce this effect.

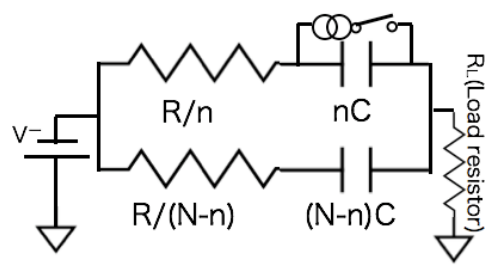

Figure 6: An equivalent circuit of MPPC. R shows quenching resistor of one pixel, $\mathrm{C}$ shows capacitance of one pixel and $n$ shows the number of pixels fire and $\mathrm{N}$ shows total number of MPPC pixels.

\subsection{Isolation by head-amplifier}

One possible way to alleviate this pulse widening effect is to isolate each MPPC using head amplifiers. Below we describe the results of our studies along this direction. Fig. 8 shows the design of head-amplifier. First, each MPPC is connected a grounded-base amplifier. Then they are summed up, and amplified by 10. Fig. 9 and Fig.10 show the response and linearity of the amplifier for impulse inputs. From these figures we conclude head-amplifier correctly sum up input pulses. Fig.11 shows the pulse shape of single MPPC input while Fig. 12 the pulse shape of 4 MPPCs inputs and Fig. 13 the pulse shape of 4 MPPCs connected as Fig. 3. From these figures we conclude the pulse widening effect is reduced to a negligible level.

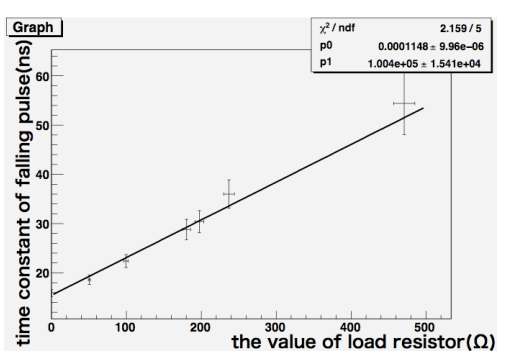

Figure 7: correlation between the value of load resistor and decay constant of MPPCs.

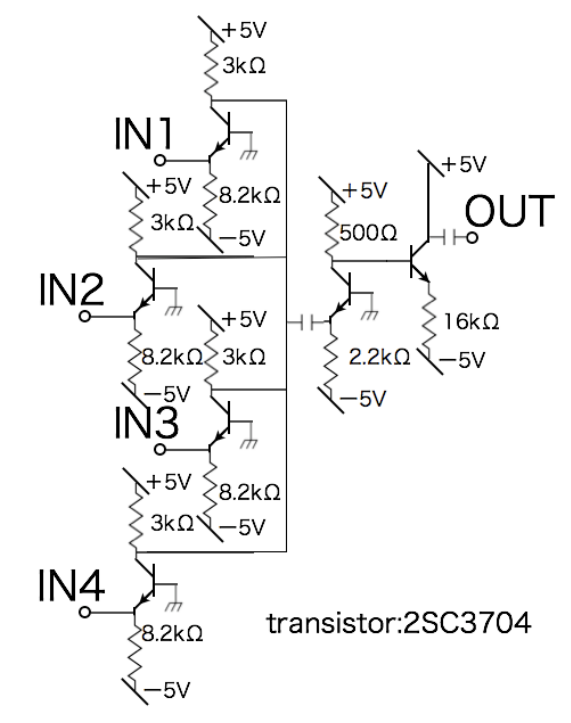

Figure 8: A circuit diagram of the head amplifier. It consists of 4 base-grounded amplifiers to isolate MPPCs each other, one base-grounded amplifier to sum up MPPC's signal, and an emitter follower (buffer). 


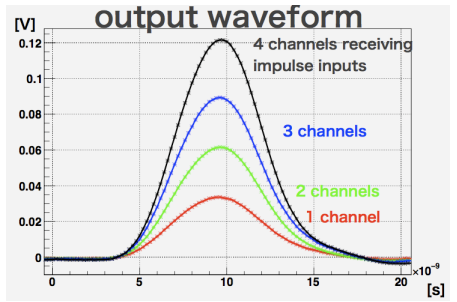

Figure 9: The pulse shape of output with impulse input.

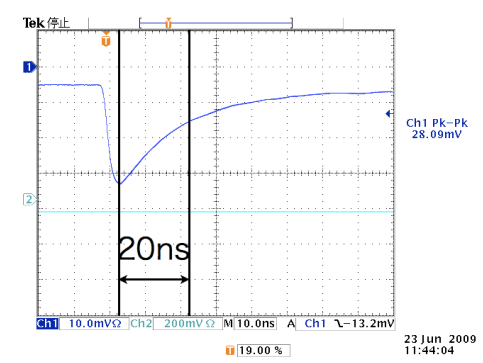

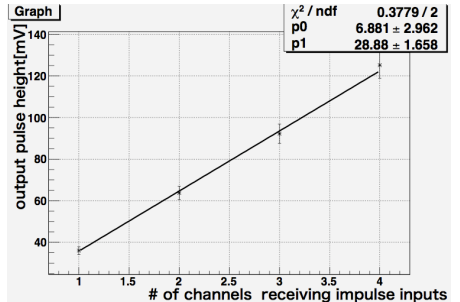

Figure 10: Correlation between \# of channel receiving impulse inputs and pulse height of output.

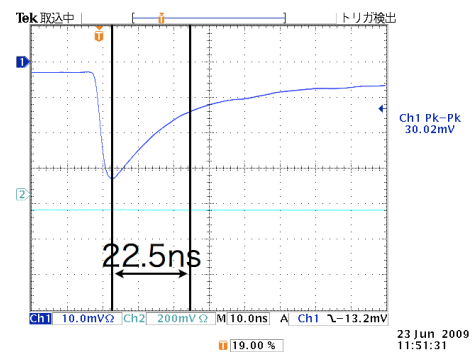

Figure 11: output waveform when only one channel Figure 12: output waveform when 4 channels receivreceiving MPPC input.

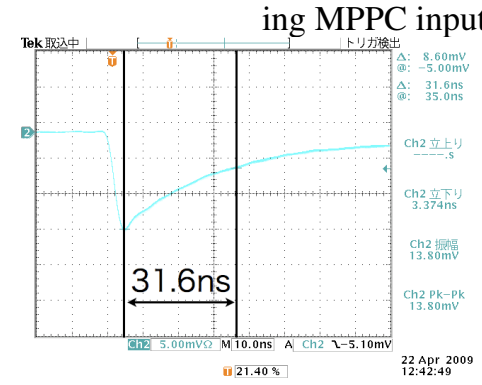

Figure 13: output waveform when not use head-amplifier and 4 MPPCs are connected in parallel as Fig. 3.

\section{Conclusion}

We have tested a parallel-connected scheme for PPD readout. The merit of this scheme is to reduce number of readout channels. In our measurement using MPPCs, we found the pulse width becomes wider when MPPCs are simply connected in parallel, as the number of parallel connec -ted MPPC increases. This phenomenon is explained by a model which takes into account the capacitance of spectator pixels. In order to alleviate the effect we developed a head-amplifier which isolates each MPPC channel. With this new read out scheme, we could reduce the pulse widening effect to a negligible level.

\section{References}

[1] J. Comfort et al. (J-PARC E14 Collab), " Proposal for $K_{L^{-}}>\pi^{0} v \bar{v}$ Experiment at J-PARC” , (2006).

[2] Hamamatsu Photonics K.K. http://jp.hamamatsu.com/products/sensor-ssd/4010/index_en.html

[3] F. Corsi, C. Marzocca et al., "Electrical Characterization of Silicon Photo-Multiplier Detectors for Optimal Front-End Design ” , 2006 IEEE NSS Conference Record, pp. 1276-1280, 